\title{
Measuring Picosecond Flashover in Closing Coaxial Spark Gap Switch
}

\author{
J. KLÜSS AND J. HÄLLSTRÖM \\ Department of Electrical and Communications Engineering, Helsinki University of Technology \\ P.O. Box 3000, FI-02015 TKK, Finland
}

\begin{abstract}
Flashover voltage and picosecond risetime of breakdown in pressurized $\mathrm{SF}_{6}$ gas is studied on a coaxial line. Gas pressure is varied from 1 to 19 bar. The homogeneous field gap has an opening from ca. 0.2 to $0.9 \mathrm{~mm}$, and flashover voltages reach $120 \mathrm{kV}$. Measurements are performed using a D-dot probe with 16 to $20 \mathrm{GHz}$ real-time oscilloscopes and a $50 \mathrm{GHz}$ sampling oscilloscope. Measured risetimes are down to ca. $50 \mathrm{ps}$, and the dependence of voltage collapse on gas density and electric field between the electrodes is reported. Integrity of data is analyzed and experimental results are compared with present theory found in literature and previous results measured with alternative real-time systems.
\end{abstract}

PACS numbers: 51.50.+v, 52.70.Gw, 52.80.-s

\section{Introduction}

Ever-growing advances in measurement technology have improved the accuracy of measuring fast transients enabling real-time sampling of picosecond pulses. Here, previous real-time (single-shot) data from digital signal analysers of lower bandwidth are compared with greater resolution series sampling data. Using alternative systems and measuring technique aims at distinguishing the phenomenon pertaining to the immensely fast voltage collapse from the characteristics of the test setup.

\section{Measurement setup}

The spark gap is of coaxial structure with identical hemispherical stainless steel electrodes facing each other (Fig. 1). The impedance of the coaxial line is maintained at $c a .30 \Omega$ along the entire coaxial structure via a biconical design. The inter-electrode distance can be adjusted and the electrodes are situated in a gas-tight chamber where the insulating gas can be pressurized accordingly. A high voltage impulse with steepness ranging from 400 to $1200 \mathrm{kV} / \mu \mathrm{s}$ is fed to one electrode while the other electrode is connected to the ground via resistance approximately equal to the characteristic impedance of the line. The two D-dot probes (standard straight bulkhead SMA jack) are located on the coaxial line $c a .7 \mathrm{~cm}$ away from the gap on both directions and inserted flush to the inner wall of the outer conductor. One probe measures the signal propagating towards the resistive load and the other the signal propagating back towards the impulse generator. Impulses were fed at $2 \mathrm{~Hz}$ and 500 samples were collected to compile a pulse with a time resolution of 2 ps.

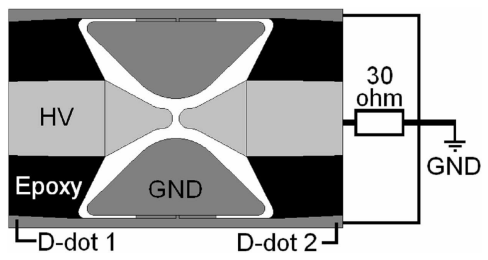

Fig. 1. Spark gap construction.

A derivative of voltage $(\mathrm{d} V / \mathrm{d} t)$ is measured and in order to calculate voltage collapse the acquired pulse is integrated. Bandwidth was improved from earlier setups [1] by implementing a Tektronix TDS8200 series sampling oscilloscope with a 80E01 sampling module having bandwidths exceeding $50 \mathrm{GHz}$. Due to the statistical nature of breakdown, disturbances which further increase data scatter and zero-level fluctuations were minimized by employing rigorous electromagnetic shielding of measurement equipment and proper grounding technique in efforts to preserve data integrity.

\section{Results}

Three sub-millimeter gap spacings were used and applied pressure increased from 1 to 19 bar. Breakdown voltages ranged from $10 \mathrm{kV}$ to $120 \mathrm{kV}$ and followed a somewhat linear relationship with pressure as described by theory. Figure 2 shows the newly acquired data $(50 \mathrm{GHz})$ along with prior results measured with alternative setups. Significant deviations from Paschen's law at lower values of $p d$ were observed which correspond to $E / p$ values exceeding approximately $15 \mathrm{kV} /(\mathrm{mm}$ bar). Here, 
$E$ is electric field strength in $\mathrm{kV} / \mathrm{mm}, p$ is pressure in bar and $d$ is gap distance in $\mathrm{mm}$.

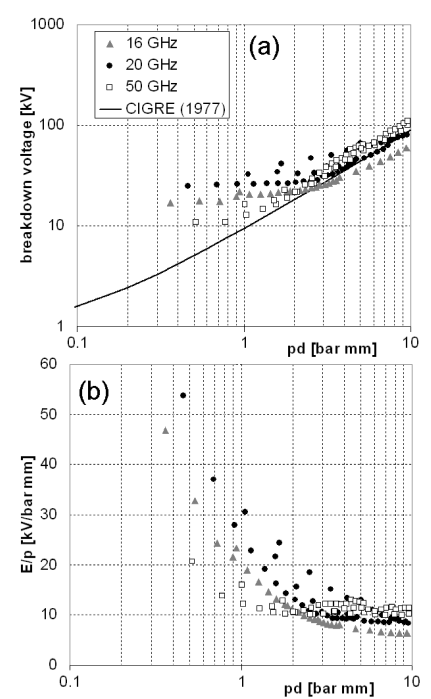

Fig. 2. Top - measured breakdown voltages plotted along the Paschen's curve [2]. Bottom $-E / p$ plotted against $p d$ values.

For each gap spacing the test switch was opened and cleaned to avoid contaminating the $\mathrm{SF}_{6}$ insulation gas. Considerable scatter was observed in breakdown voltages and measured pulse shapes for the first 1000 triggers. As the series sampling oscilloscope collects a number of samples from which it builds a pulse, this scatter significantly hindered measurements. It was necessary to trigger the test switch at least 1000 times until subsequent pulses stabilized and scatter was decreased to an acceptable level. Malik and Qureshi [3] refer to this as the "conditioning effect" where weak points of the gap are destroyed by breakdown sparks.

Two distinct $\mathrm{d} V / \mathrm{d} t$ pulse shapes were observed which allows the measured processes to be grouped into three categories - region I, transition region, and region II which are evaluated in Table. Calibration of the D-dot probes [4] was not consistent for the entire measurement range. Hence, presented $\mathrm{d} V / \mathrm{d} t$ values are arbitrary. However, these values do not effect the calculation of risetimes which were conducted from normalized $10 \%$ to $90 \%$ coordinates.

TABLE

$\mathrm{d} V / \mathrm{d} t$ pulse regions.

\begin{tabular}{c|c|c|c}
\hline \hline Region & $\begin{array}{c}\text { Pressure range } \\
{[\mathrm{bar}]}\end{array}$ & $\begin{array}{c}E / p \\
{[\mathrm{kV} /(\mathrm{mm} \text { bar })]}\end{array}$ & $\begin{array}{c}\text { Risetime } \\
{[\mathrm{ps}]}\end{array}$ \\
\hline I & $1-3$ & $>30$ & 50 \\
Transition & $4-9$ & $10-30$ & N $/ \mathrm{A}$ \\
II & $>10$ & $\sim 10$ & $200-350$
\end{tabular}

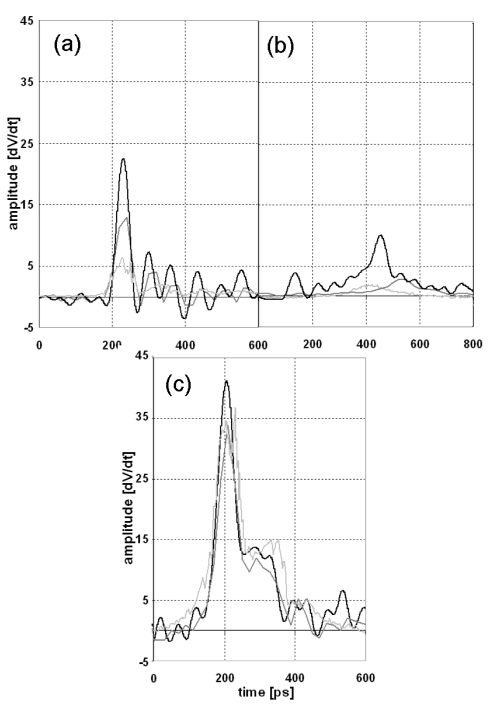

Fig. 3. Comparison of $\mathrm{d} V / \mathrm{d} t$ pulse shapes where light grey is from $50 \mathrm{GHz}$ series sampling, black is $20 \mathrm{GHz}$ Tektronix DSA72004 using interpolation and dark grey is $16 \mathrm{GHz}$ Tektronix without interpolation. Gap distance $d \sim 0.50 \mathrm{~mm}$ and pressure is (a) $p=1$ bar, (b) $p \sim 5$ bar, (c) $p=19$ bar.
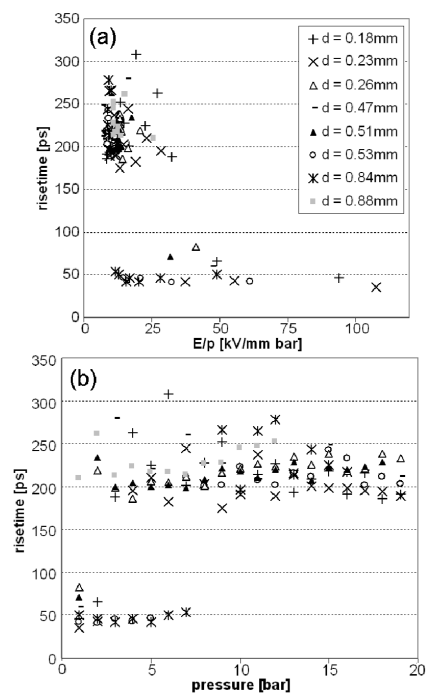

Fig. 4. Risetime of measured voltage collapse correlated with (a) $E / p$ and (b) pressure.

Region I, where $E / p$ exceeds $30 \mathrm{kV} /$ (mm bar), is characterized by a clean $\mathrm{d} V / \mathrm{d} t$ pulse with a distinct rising face and peak which returns to zero level, as shown in Fig. 3a, thus giving a linear voltage collapse from which risetimes are easily calculated. When pressure is increased, which in turn decreases $E / p$ values, a transition region begins where the measured $\mathrm{d} V / \mathrm{d} t$ pulse becomes almost unrecognizable and differing with each trigger and consequently risetime calculations are misleading (Fig. 3b). Deviations from Paschen's law described ear- 
lier fall into region $I$ and continue into this transition region. Once pressure is increased so that $E / p$ is approximately $10 \mathrm{kV} / \mu \mathrm{s}$, a repetitive pulse is once again observed (Fig. 3c).

However, this pulse after peaking does not directly return to zero level but rather plateaus, signifying that the gradient of the voltage collapse changes and thus increases risetimes as calculated in Fig. 4.

\section{Conclusions}

This paper presents short $\mathrm{SF}_{6}$ gap risetime measurements performed with a $50 \mathrm{GHz}$ series sampling oscilloscope and confirms results acquired with lower bandwidth systems. The changes in the observed pulse shapes suggest an alteration in the breakdown phenomenon within different pressure ranges corresponding to certain $E / p$ values with relatively constant risetime. Furthermore, deviation from Paschen's law is also restrained to $E / p$ values observed in region I and the transition region.
Since gap distance affects field strength, variations in $d$ appear to somewhat influence the onset of each region.

\section{Acknowledgments}

Research financed by Fortum Foundation and Tektronix TDS8200 courtesy of the Finnish Defence Forces Technical Research Center.

\section{References}

[1] J. Klüss, M. Sc. Thesis, Helsinki University of Technology, 2007.

[2] CIGRE, Breakdown of Gases in Uniform Fields, Working Group 15.03 (1977).

[3] N.H. Malik, A.H. Qureshi, IEEE Trans. Electr. Insul. 13, 135 (1978).

[4] S. Burkhart, IEEE Trans. Microwave Theory Techniques 33, 262 (1985). 